BMJ Health \& Care Informatics

\title{
Outcomes of a feasibility trial using an innovative mobile health programme to assist in insulin dose adjustment
}

\author{
Anish Menon (D) , ${ }^{1,2}$ Farhad Fatehi (D) , ${ }^{1,3}$ Hang Ding, ${ }^{4}$ Dominique Bird, \\ Mohan Karunanithi, ${ }^{4}$ Leonard Gray, ${ }^{1}$ Anthony Russell ${ }^{1,2}$
}

To cite: Menon A, Fatehi F, Ding $\mathrm{H}$, et al. Outcomes of a feasibility trial using an innovative mobile health programme to assist in insulin dose adjustment. BMJ Health Care Inform 2019;26:e100068. doi:10.1136/ bmjhci-2019-100068

Received 01 June 2019 Revised 08 October 2019 Accepted 12 October 2019

\section{Check for updates}

(c) Author(s) (or their employer(s)) 2019. Re-use permitted under CC BY-NC. No commercial re-use. See rights and permissions. Published by BMJ.

${ }^{1}$ Centre for Health Services Research, University of Queensland, Brisbane, Queensland, Australia ${ }^{2}$ Department of Diabetes and Endocrinology, Princess Alexandra Hospital, Brisbane, Queensland, Australia ${ }^{3}$ School of Advanced Technologies in Medicine, Tehran University of Medical Sciences, Tehran, Iran

${ }^{4}$ The Australian EHealth Research Centre, The Commonwealth Scientific and Industrial Research Organisation, Brisbane, Queensland, Australia

Correspondence to Dr Anish Menon; anish.menon@uq.net.au

\section{ABSTRACT}

Objective Intensification of diabetes therapy with insulin is often delayed for people with suboptimal glycaemic control. This paper reports on the feasibility of using an innovative mobile health (mHealth) programme to assist a diabetes insulin dose adjustment (IDA) service.

Methods Twenty adults with diabetes referred to a tertiary hospital IDA service were recruited. They were provided with a cloud-based mobile remote monitoring systemthe mobile diabetes management system (MDMS). The credentialled diabetes educator (CDE) recorded the time taken to perform IDA utilising the MDMS versus the conventional method-which is a weekly adjustment of insulin doses by a CDE through telephone contact based on three or more daily blood glucose readings. Participants and staff completed a feedback questionnaire.

Results The CDE spent $55 \%$ less time performing IDA using MDMS than using the conventional method. The participants were satisfied with MDMS use and the CDEs reported improved efficiency.

Conclusion Incorporating a mHealth programme for an IDA service has the potential to improve service delivery efficiencies while simultaneously improving the patient experience.

\section{INTRODUCTION}

Diabetes mellitus is a chronic condition in which the risk of complications is reduced by achieving recommended blood glucose level targets. ${ }^{2}$ In type 2 diabetes, insulin is commenced if lifestyle measures and other antidiabetic agents do not achieve the glycaemic target. Insulin is commenced from diagnosis in type 1 diabetes and dosage adjustments may be required intermittently according to glycaemic control. ${ }^{3}$ Delay in initiation or intensification of insulin therapy can contribute to suboptimal glycaemic control. ${ }^{45}$ Referral to an insulin dose adjustment (IDA) service for either insulin initiation or titration can assist in improving glycaemic control, but it is a resource-intensive and time-consuming process. Previously reported studies have utilised mobile health (mHealth) strategies to improve efficiencies and achieve better glycaemic outcomes over conventional methods. ${ }^{6-8}$ Of the reported studies, most have targeted basal insulin titration (eg, long-acting insulin) but not a combination of complex insulin regimes that include basal and bolus insulins. ${ }^{78}$ Moreover, there are no reported trials in an Australian setting that employed mHealth for IDA in people with diabetes other than weekly diabetes education/coaching for type 1 diabetes. ${ }^{9}$

The IDA service has been described elsewhere. ${ }^{10}$ Briefly, a diabetes health professional, often a credentialled diabetes educator (CDE), located at a tertiary hospital diabetes centre runs the IDA service in liaison with the endocrinologist to provide education on insulin and its administration (for insulinnaïve patients) followed by weekly phone contacts for IDA advice. The patients achieve recommended glycaemic targets usually within 4-6 weeks. We designed a mobile-based IDA (mIDA) programme to improve the efficiency of the IDA service, improve the patient experience and reduce the possibility of transcription errors. We previously tested and reported on the mIDA programme through a proof-of-concept trial involving patients with diabetes and stable glycaemic control.$^{11}$ The mIDA uses the mobile diabetes management system (MDMS) comprising a smartphone app (android version for this trial) which pairs with a Bluetooth-enabled glucose meter and automatically uploads blood glucose levels (BGLs) to a clinician portal via the 'internet cloud' (figure 1). ${ }^{11}$ The patient has the option of manually entering their insulin doses (if different to the insulin prescription on the mobile app) and free-text comments with each BGL entry. The MDMS utilises cloud-based remote monitoring to present BGL and their trends on the app at the patient-end while enabling the clinicians to visualise the data on the portal synchronously and send messages and insulin dose recommendations to the patient. The home 


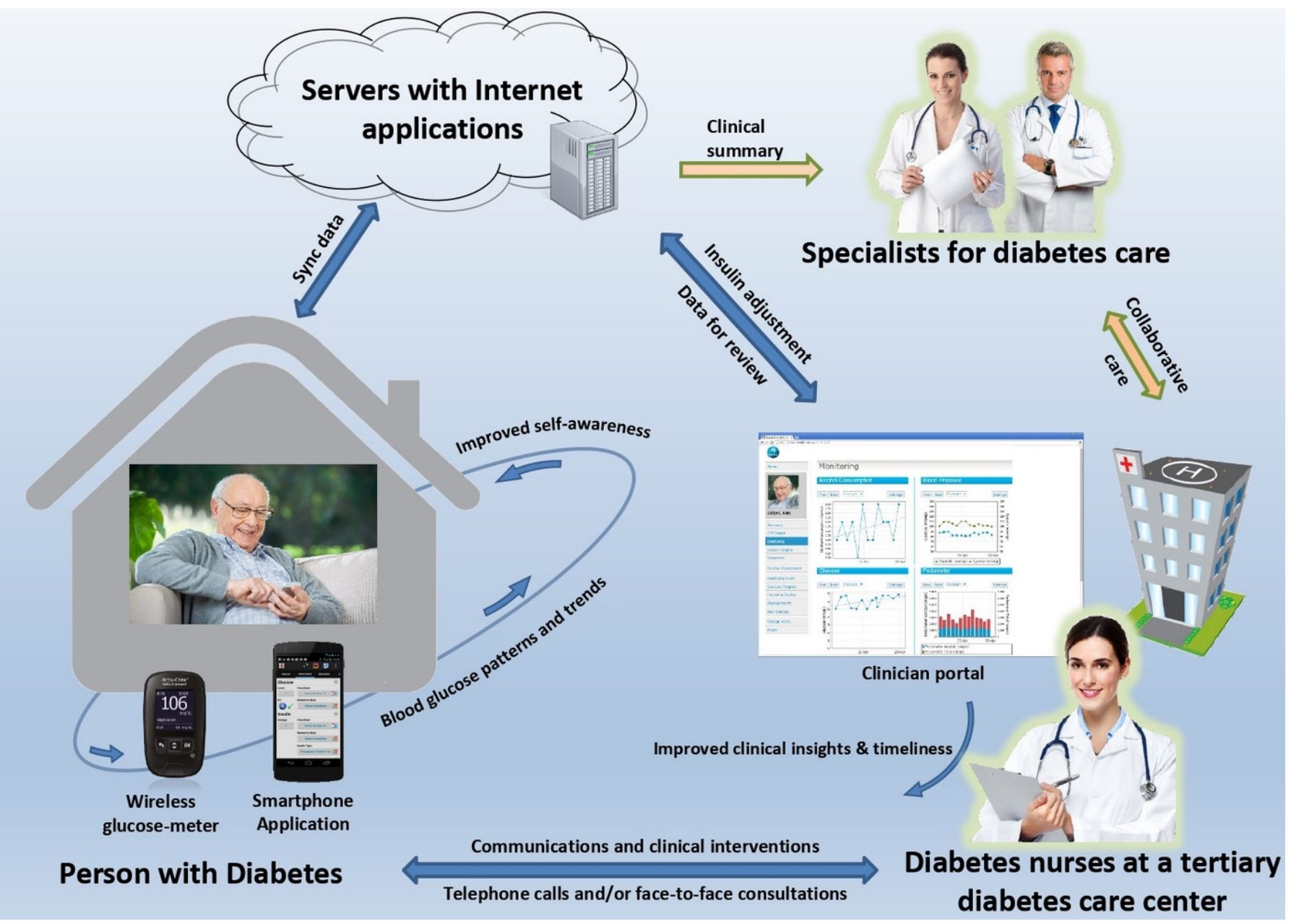

Figure 1 The care model for the insulin dose adjustment programme using the mobile diabetes management system ${ }^{11}$.

page of the portal displays a summary of each individual's glycaemic status. This is designed to help the prioritisation of patient follow-ups, and the process can be customised depending on desired outcomes and local resources.

The MDMS was developed through an iterative process with the aim to enhance the quality and timeliness of the patient-clinician interaction, and support patient self-management of diabetes. The earlier mIDA proofof-concept study showed promising levels of adherence, usability, perception of usefulness and satisfaction. ${ }^{11}$ The study participants provided feedback to improve the MDMS. The suggested changes were incorporated in the version used in this trial. This paper reports on a feasibility trial assessing the user experience of the MDMS in the mIDA programme at a tertiary hospital diabetes centre and potential improvements in the efficiency of the IDA service.

\section{METHODS}

Twenty patients referred to the IDA service from the Brisbane Princess Alexandra Hospital (PAH) diabetes outpatient clinic, PAH diabetes telehealth clinic and a PAH community outreach diabetes clinic were recruited. The inclusion criteria were as follows: $\geq 18$ years of age, patients with diabetes referred to the IDA service, minimum education level of year 6 (or equivalent) and using a smartphone. Participants were excluded if they were living in areas with no coverage of the $3 \mathrm{G} / 4 \mathrm{G}$ cellular internet or were unable to communicate in English.

Each participant recruited in the trial was provided with a Bluetooth-enabled glucose-meter (Accu-Chek Aviva Connect meter, Roche Diagnostics GmbH, Basel, Switzerland). If a participant's smartphone was incompatible with the app and/or the glucose-meter, the participant was provided with a compatible smartphone. The CDEs trained each participant in-person on the use of the app and the glucose-meter. The CDEs then followed up participants to titrate the insulin dose once or twice weekly according to their glycaemic variability. For each interaction, the CDEs initially used the MDMS to make the necessary IDA, send a text-message to the participant via the portal and then record the total time taken. The text-message contained insulin dose recommendations and/or other advice regarding improving their glycaemic status. Immediately following this, they contacted the participants via conventional method over the phone, provided IDA advice and then recorded the time taken via this method. If there were technical issues that could 
not be fixed by the CDEs, a software-engineer who had assisted in the development of MDMS was engaged.

On discharge from the IDA service, participants were asked to fill a Likert-scale questionnaire to assess the user experience. Likert scales were scored as (1) strongly disagree, (2) disagree, (3) neutral, (4) agree and (5) strongly agree. This questionnaire was previously used in the earlier proof-of-concept trial. The participants were able to enter free-text comments on what they liked and what they disliked, and on further improvements to the MDMS. Feedback from the CDEs was obtained by an online questionnaire.

Statistical analysis was done using SPSS (IBM Corp. Released 2017. IBM SPSS Statistics for Windows V.25.0). Data are presented in percentages, means and medians. Mann-Whitney U test or Student t-test was used as appropriate.

\section{RESULTS}

The median age of the 20 participants recruited to the study was 58 years (ranging from 20 to 69 years, IQR $65-42$ ) and $45 \%$ were female. Two participants were lost to follow-up as they stopped accessing the diabetes service and were uncontactable. All participants had type 2 diabetes except one with type 1 diabetes. The average training time in use of the MDMS was $45 \mathrm{~min}$. The median IDA service duration was 3 weeks with a range from 1 to 14 weeks.

There was a total of 90 attempted IDA contacts by the CDE for 18 participants. There were 67 instances when both the MDMS and conventional IDA delivery times were recorded. The mean time for IDA using MDMS was $5.1 \mathrm{~min}$ (SD 3.1), a significant time saving compared with $11.3 \mathrm{~min}$ (SD 6.0) with conventional approaches ( $\mathrm{p}$ value $<0.001)$. Overall, there were five instances where instead of MDMS, only conventional care time taken was recorded for providing the IDA service. The reasons for this included technical issues on two occasions, participant requiring hypoglycaemic management education, attendance at the outpatient clinic unrelated to diabetes and discharge from the IDA. On 17 (18.9\%) occasions, participants were not contactable. Of these occasions, the time taken for conventional contact was recorded on 10 occasions. The time taken ranged from 2 to $15 \mathrm{~min}$, with a median of $5 \mathrm{~min}$ (IQR 10-4). The higher time requirements were more often related to the process involved in making multiple attempts to contact participants. Out of the 17 non-contactable instances, IDA times were recorded for the MDMS on 15 occasions with a median time of 5 min (ranging from 1 to 10 min IQR 5-1).

There were MDMS-related technical issues (both participant and provider-related) documented by the CDEs in $6(6.7 \%)$ out of the 90 contacts. These included issues with Bluetooth-pairing, compatibility of the app with the smartphone's operating system and inability to send textmessages through the MDMS.
There was a single instance of an error in BGL out of the few hundred readings relayed to the CDE over the phone which was picked up on comparison with the BGL uploaded to the clinician portal.

User experience data were available for 13 out of the 18 participants (figure 2 ). The participants were satisfied with the MDMS and had a high preference for continuing to use the system as indicated by the score of 4.7 out of 5 . They disagreed that participation in the study took too much time. There were technical problems related to the automatic Bluetooth-transfer of BGL. However, the mean score of 3.6 was an improvement on the previous proof-of-concept study's mean score of 2.6 in response to 'I had no technical problem using the system'. The free-text comments also highlighted that the participants liked the visual representation of data, the increased access to healthcare providers and the ease of use. Some of the participants' quotes highlight these aspects-'the graph sections, seeing my patterns of my BGL gave me a visual take on what's going on with me and make those changes', 'less appointments and more feedback from the health team to assist getting on track' and 'easy to use'. The participants' feedback recommended to enhance the Bluetooth connectivity, usability of the app screens and the provision of technical assistance when faced with technology-related issues. Review of usage data of the 18 participants who completed the study showed that a median of 99 BGL entries per participant was uploaded in 4 weeks (IQR - 114 - 72). However, only five participants entered their insulin doses. The experience from the four CDEs who participated in the trial was positive, with three out of four reporting no problems and stating that the MDMS improved their efficiency. The CDEs questionnaire free-text comments suggested improvements to the MDMS, such as an easier set-up for patients and clinicians, and a confirmatory message if the patient has seen the advice sent to them.

\section{DISCUSSION}

In this feasibility trial, we evaluated the time-efficiencies and user experience of a newly developed mIDA service, following promising user feedback from an earlier proof-of-concept trial. ${ }^{11}$ The findings suggest that mIDA improves time-efficiencies by $54.8 \%$ from a CDE perspective, has the potential to reduce transcription error and improves the patient experience in the IDA service. This trial is part of a series of projects aimed at testing a new model of specialist outpatient diabetes care using various eHealth strategies.

The IDA service at the PAH has a capacity of accommodating 50 new diabetes patients per week. From the median time taken per IDA contact in this study and using a conservative estimate of 1 contact per patient per week, the total calculated time per week required for IDA using MDMS is $255 \mathrm{~min}$ as opposed to $565 \mathrm{~min}$ with the conventional IDA service. This equates to a potential CDE time saving of 5.16 hours/week. A previous cloud-based insulin 
Participation in the study took too much time

Overall, I am satisfied with the mobile-based system

If it was possible I would continue using this system

Using the system gave me confidence that I could better manage my diabetes

I took the advice sent via mobile application seriously

My family were supportive of me using the system

I had no technical problem using the system

The instruction on how to use the system were sufficient

The mobile application was easy to use

I could easily use the mobile phone to upload the results

I could easily use the glucose-meter to send the results
1.6
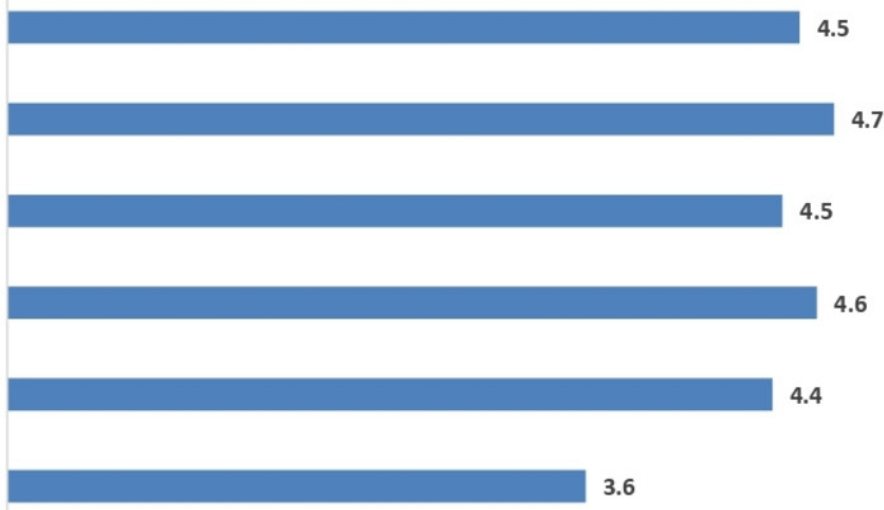

4.4
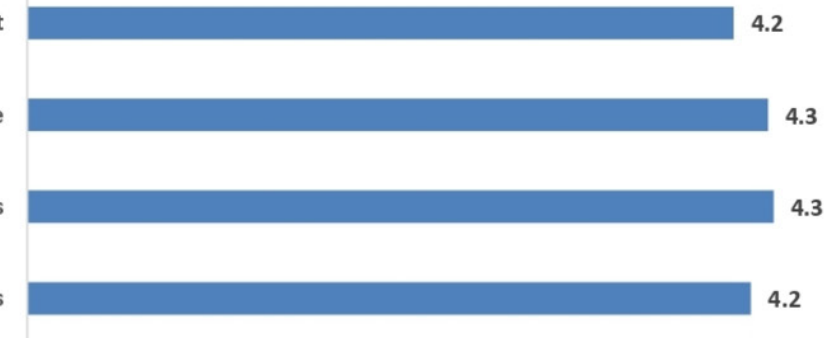

Figure 2 Scores of the questionnaire to evaluate the user experience.

titration study with 40 participants examining basal insulin initiation and titration had reported a greater time savings of $15.7 \mathrm{~min}$ per subject in the intervention arm. ${ }^{7}$ This would equate to a CDE time saving of 13.08 hours/ week if this were to be replicated at the PAH IDA service. This difference in time savings are likely to be due to the within-subject design and inclusion of more complex participants (on both basal and bolus insulins) in our trial. Similarly, pregnant women with gestational diabetes, which like IDA requires frequent healthcare professional contact, had improved blood glucose control by using an app and required two less clinic visits on average during their pregnancy compared with non-users of the app. ${ }^{12}$

Phone contact requires synchronous communication and this study showed that a substantial proportion of patients $(18.9 \%)$ were not readily available via phone contact. The mIDA programme can provide valuable feedback to patients through easier communication methods like text-messaging or in-app messages. The time savings are likely to be higher than reported in this feasibility trial, as the CDEs first used the MDMS system followed by the conventional approach for the same patient. Moreover, with regular MDMS use, the CDEs are likely to become quicker in navigating the system. Time taken for technical training of participants has not been considered in these calculations. This is because we foresee that in-person technical training can be replaced by patient codesigned step-by-step training videos, that the use of technology like mobile apps outside of a healthcare setting will become more widespread, and that the same individual might require multiple occasions of IDA service in their diabetes journey. Thus, we see that MDMS use provides the 'gift of time', enhancing the ability of the CDEs to see more patients and provide a high-quality service leading to better user experience for people with diabetes. $^{12}$

Transcription errors are a significant concern when using conventional methods to deliver IDA. Although there was only a single instance of error, this could have led to potentially serious consequences had the error missed a documented hypoglycaemic episode and inadvertently resulted in increased insulin doses. ${ }^{13}$

The patient feedback questionnaire confirmed the earlier favourable proof-of-concept study scores on ease of use, the time required to use, and overall satisfaction with the MDMS along with helping the participants to be more confident in self-managing their diabetes. ${ }^{11}$ However, only a small proportion of participants entered their insulin doses as compared with BGL. Automation, such as the use of cloud-connected insulin pen devices, is likely to improve clinician access to data. ${ }^{14}$ Although the participants continued to have a few Bluetooth connectivity issues between the app and the glucose-meter, the majority of participants 
agreed that there were no technical problems using the system; this was an improvement on the proof-ofconcept trial. With technological advances, this is not unexpected and is likely to improve with time. ${ }^{15}$ The CDEs provided feedback on patient safety measures and advised that displaying on the clinician portal a record of the patients accessing the clinicians' message would be useful. This will help to close the communication loop and save time by avoiding further confirmatory patient follow-ups via phone.

As with the mobile insulin titration intervention study, we are now transitioning the mIDA programme to an implementation trial for understanding generalisability and acceptability among patients and providers. ${ }^{16}$ As technology advances, innovative solutions like patient facing-apps/devices that guide self-titration of insulin through automated prompts with support from healthcare providers will become increasingly available and might be even more cost-effective. ${ }^{6}$

Limitations of the trial include the small sample size. However, this was a feasibility trial with double the sample size from the earlier proof-of-concept trial. Reported literature suggests a number as low as 10 has been used in feasibility trials. ${ }^{17}$ Well-designed randomised controlled trials (RCTs) are required to compare the MDMS intervention versus conventional care, but we opted for a feasibility trial before an RCT based on a framework for telehealth interventions. ${ }^{18}$ Given our findings it is reasonable to hypothesise that MDMS could save time as in our trial the CDEs used MDMS first followed by conventional care. However, a possibility is that some of the conventional interactions might have helped better educate the participant regarding diabetes self-management, which might not be possible through text-messages alone. Hence, the mIDA programme might require a hybrid approach - a combination of regular text-messaging and occasional phone contacts if required. As the participants received both MDMS and conventional care, caution needs to be exercised in interpreting the user experience findings, especially in relation to the overall satisfaction, continued system use and self-management confidence. However, the MDMS intervention is a radical departure from conventional practice and the rest of the questions were specific to the MDMS intervention. The user experience questionnaire, though an unvalidated one, enabled the comparison of responses across the proof-of-concept and feasibility trials. Moreover, there were no highly reliable usability questionnaires specifically designed for mHealth apps at the time of conducting the trial. ${ }^{19} \mathrm{We}$ did not report on adherence as it would be impossible to differentiate between the two approaches given a withinsubject design. mHealth intervention trials have shown that the users improve glycaemic status in the short-term (up to 12 months) ${ }^{20}$ but reports regarding adherence are mixed and difficult to evaluate. ${ }^{11} 21$ As the IDA process usually lasts for a period of 4-6 weeks only, the service efficiency derived from mHealth strategies are likely to be sustainable.
We hope the findings of this feasibility trial lead to further research examining the cost-effectiveness of mHealth tools in assisting the IDA service. mHealth solutions like the mIDA programme have the potential to help design new models of care and build capacity to serve a wider cohort of patients within existing resources. Following this feasibility trial, our next step is to test a new model of outpatient specialist diabetes care that aims to redesign the current in-person visits for specialist care through the provision of better self-management support outside of clinic visits through enhancements to the MDMS such as contextual automated text-messages based feedback, periodic patient online self-report and provision of virtual clinics. The protocol of this pilot RCT has been published. ${ }^{22}$

\section{CONCLUSION}

The mIDA has the potential to improve IDA service efficiency and user experience. We believe it is important that we improve diabetes care delivery efficiencies given the increasing prevalence of diabetes and workforce shortages. A mHealth programme for IDA service might assist in addressing the therapy intensification inertia in diabetes clinical practice.

\section{Twitter Anish Menon @AnishMenon18 and Farhad Fatehi @farhadfatehi}

Acknowledgements The authors acknowledge the participants of the trial and the clinical staff at the Department of Diabetes and Endocrinology, Princess Alexandra Hospital for facilitating this research. AM is a recipient of Diabetes Queensland Scholarship. AM and DB are financially supported by the Centre of Research Excellence in Telehealth funded by the National Health and Medical Research Council (NHMRC; grant ID: APP1061183). FF is financially supported by the Queensland Government through an Advance Queensland Research Fellowship. We acknowledge Roche Australia for the provision of glucose-meters.

Contributors All authors contributed to the paper.

Funding This project is funded by a grant from the Telehealth Seed Funding, Queensland Health, and Metro South Health, Queensland Health.

Competing interests None declared.

Patient consent for publication Not required.

Ethics approval The trial received ethics approval from the Metro South Human Research Ethics Committee, Brisbane, Queensland, Australia (Ref: HREC/14/ QPAH/686).

Provenance and peer review Not commissioned; externally peer reviewed.

Open access This is an open access article distributed in accordance with the Creative Commons Attribution Non Commercial (CC BY-NC 4.0) license, which permits others to distribute, remix, adapt, build upon this work non-commercially, and license their derivative works on different terms, provided the original work is properly cited, appropriate credit is given, any changes made indicated, and the use is non-commercial. See: http://creativecommons.org/licenses/by-nc/4.0/.

\section{ORCID iDs}

Anish Menon http://orcid.org/0000-0002-8176-0292

Farhad Fatehi http://orcid.org/0000-0001-9888-1966

\section{REFERENCES}

1 Holman RR, Paul SK, Bethel MA, et al. 10-Year follow-up of intensive glucose control in type 2 diabetes. $N$ Engl J Med Overseas Ed 2008;359:1577-89.

2 Nathan DM, Group DER, For the DCCT/EDIC Research Group. The diabetes control and complications trial/epidemiology of diabetes 
interventions and complications study at 30 years: overview. Diabetes Care 2014;37:9-16.

3 Fonseca VA. Defining and characterizing the progression of type 2 diabetes. Diabetes Care 2009;32:S151-6.

4 Evans ML, Golubic R. Technology to overcome clinical inertia in insulin therapy. The Lancet 2019;393:1078-80.

5 Khunti K, Millar-Jones D. Clinical inertia to insulin initiation and intensification in the UK: a focused literature review. Prim Care Diabetes 2017;11:3-12.

6 Bergenstal RM, Johnson M, Passi R, et al. Automated insulin dosing guidance to optimise insulin management in patients with type 2 diabetes: a multicentre, randomised controlled trial. The Lancet 2019;393:1138-48.

7 Hsu WC, Lau KHK, Huang R, et al. Utilization of a cloud-based diabetes management program for insulin initiation and titration enables collaborative decision making between healthcare providers and patients. Diabetes Technol Ther 2016;18:59-67.

8 Levy N, Moynihan V, Nilo A, et al. The mobile insulin titration intervention (MITI) for insulin adjustment in an urban, low-income population: randomized controlled trial. J Med Internet Res 2015:17:e180.

9 Kirwan M, Vandelanotte C, Fenning A, et al. Diabetes selfmanagement smartphone application for adults with type 1 diabetes: randomized controlled trial. J Med Internet Res 2013;15:e235.

10 Menon A, Gray L, Fatehi F, et al. Mobile-based insulin dose adjustment for type 2 diabetes in community and rural populations: study protocol for a pilot randomized controlled trial. Ther Adv Endocrinol Metab 2019;10:2042018819836647.

11 Ding H, Fatehi F, Russell AW, et al. User experience of an innovative mobile health program to assist in insulin dose adjustment: outcomes of a proof-of-concept trial. Telemed J E Health 2018;24:536-43.

12 The Topol review preparing the healthcare workforce to deliver the digital future: health education England, 2019. Available: https:// topol.hee.nhs.uk/
13 Sowan AK, Vera A, Malshe A, et al. Transcription errors of blood glucose values and insulin errors in an intensive care unit: secondary data analysis toward electronic medical Record-Glucometer Interoperability. JMIR Med Inform 2019;7:e11873-e.

14 Bailey TS, Walsh J, Stone JY. Emerging technologies for diabetes care. Diabetes Technol Ther 2018;20:S2-78-S2-84.

15 Heinemann L. Future of diabetes technology. J Diabetes Sci Technol 2017; $11: 863-9$

16 Levy NK, Orzeck-Byrnes NA, Aidasani SR, et al. Transition of a TextBased insulin titration program from a randomized controlled trial into real-world settings: implementation study. J Med Internet Res 2018;20:e93-e.

17 Billingham SAM, Whitehead AL, Julious SA. An audit of sample sizes for pilot and feasibility trials being undertaken in the United Kingdom registered in the United Kingdom clinical research network database. BMC Med Res Methodol 2013;13:104.

18 Fatehi F, Smith AC, Maeder A, et al. How to formulate research questions and design studies for telehealth assessment and evaluation. J Telemed Telecare 2017;23:759-63.

19 Zhou L, Bao J, Setiawan IMA, et al. The mHealth APP usability questionnaire (MAUQ): development and validation study. JMIR Mhealth Uhealth 2019;7:e11500-e.

20 Kitsiou S, Paré G, Jaana M, et al. Effectiveness of mHealth interventions for patients with diabetes: an overview of systematic reviews. PLoS One 2017;12:e0173160-e.

21 Shan R, Sarkar S, Martin SS. Digital health technology and mobile devices for the management of diabetes mellitus: state of the art. Diabetologia 2019;62:877-87.

22 Menon A, Fatehi F, Bird D, et al. Rethinking models of outpatient specialist care in type 2 diabetes using eHealth: study protocol for a pilot randomised controlled trial. Int J Environ Res Public Health 2019;16:959. 